\title{
ANTIBIOTICS: A FOOD SAFETY ISSUE
}

\author{
Alina Stancu ${ }^{1}$, Nicolae Suvorov ${ }^{2}$
}

\begin{abstract}
Population growth and globalization are currently having many negative effects on the agri-food sector's ability to ensure safe and qualitative food. Use of medicines and chemicals in animal husbandry can considerably increases production and animals' resistance to disease and pests. Elevated levels of exposure to food contaminated with chemical residues from the use of antibiotics in animal husbandry and various growth hormones are very dangerous to human health. They contribute to genetic changes at the cellular level and decreased resistance of the body to viruses and bacteria. Frequent use of antibiotics may result in chemical residues in milk, meat, eggs and honey due to large-scale application of drugs in veterinary practice. In addition to its toxicity, antibiotic residues are carcinogenic and ingested in the long term lead to increased tolerance to medication against human diseases caused by deadly bacteria. Continuous monitoring of the production phase of the agri-food chain is absolutely necessary to identify the risks of contamination and reduce the use of illegal antibiotics in animal husbandry. This research aims to determine the main causes of food contamination with chemical residues and to create an overview of the negative effects that antibiotics have on human health.
\end{abstract}

Key words: antibiotics, chemical residues, agri-food chain, food safety.

JEL'3 : I10, L66, Q18

\section{Introduction}

Food safety and human exposure to chemical residues from the irresponsible use of chemicals in agriculture is frequently debated issue worldwide. More and more people are interested in how animals are raised, as animal-based antibiotics and pesticides in crops pose serious risks of contamination, especially if they exceed the limits approved by the World Health Organization (WHO, 2019). Although the

1 Alina Madalina Stancu, Ph.D. Student, the Bucharest University of Economic Studies, Piata Romana no. 6, 010374 Bucharest, Romania, Phone: +40 735361 478, E-mail: stancualina13@stud.ase.ro

2 Nicolae Suvorov, Ph.D. Student, (corresponding author), the Bucharest University of Economic Studies, Pata Romana no. 6, 010374 Bucharest, Romania, Phone: +40 740474 653, E-mail: suvorov.nicolae@gmail.com

3 Article info: Review Article, Received: $8^{\text {th }}$ December 2020, Accepted: $13^{\text {th }}$ December 2020. 
1940 was marked by the advent and use of penicillin as an antibiotic, mentioned chemical substances have a long history as they have being used for centuries by ancient Egyptians, Chinese and Native Americans who applied different types of mould to treat wounds and various infections. Today, antibiotics are an effective way of treating infections with pathogenic bacteria, while they are widely used in the medical industry and animal husbandry. Due to the irresponsible use of antibiotics in the preparatory industries, the Centres for Disease Control and Prevention (CDC) have raised concerns regarding the negative effects on human health. Recent analysis (CDC, 2013) concluded that almost half of prescriptions are unnecessary. If antibiotics and drug treatments are used responsibly and truthfully in livestock and poultry production, they could be effective tools that lead to the ability to ensure global food security, increase GDP and at the same time regulate prices so that the product market of meat avoid facing of economic crisis. Problems occur when they are used too often and cause the appearance of antibiotic residues in food consumed by human population. So, increasing the risk of contamination and proliferation of bacteria, what is linked to the use of a considerable amount of antibiotic, can increase resistance and lead to appearance of more infectious pathogenic microorganisms.

The livestock sector has been marked since 1940 by the appearance and application of penicillin as an antibiotic in order to increase the resistance of animals to diseases, playing an important role in both human and animal health. Even if the administration of antibiotics in animal husbandry brings positive effects in terms of monitoring, prevention and control of infectious diseases, they cause the appearance of chemical residues in final products and their ingestion by human population increases the risk of developing more resistant bacteria (Aarestrup et al., 1998), leading to decreased biliary activity (Lin, 2014). Irresponsible use of antibiotics causes genetic mutations in bacteria that once become more resistant, increase in infection and can be more easily transmitted from animals to human population (Stanton, 2013). Over time, alternatives such as immunomodulatory agents, bacteriophages and their lysins and antibacterial vaccines (Millet, Maertens, 2011) have been used to minimize the risk of human exposure to chemical residues of antibiotics in meat consumed (Millet, Maertens, 2011), while in certain European countries their administration was prohibited.

Agri-food quality management is a widely debated issue worldwide in the context of streamlining the global supply chain (Kuei et al., 2011; Soltani et al., 2011). Food security is an essential indicator, highly controversial due to global scandals regarding contaminated food and shortages in mentioned sector (Roth et al., 2008; Auler et al., 2017). The presence of various contaminated agents inscribed or not on the label, whether they are chemical, physical or biological causes massive losses both in 
terms of the value of stocks and economic losses (Dani, Deep, 2010). In addition to economic losses, non-compliance with food security is a very common public health problem among the poor. Contaminated food causes 600 million diseases and 420 thousand deaths per year globally (WHO, 2019). In order to avoid these externalities as much as possible, the importance of food security in the public and private sector is widely debated in order to achieve an efficient supply chain management system (Auler et al., 2017).

The Food and Agricultural Organisation (FAO) defines the concept of food safety as the ability of a country to provide food that does not cause disease or health implications after consuming (FAO, 2003). Since 1963, the concept of food safety has been delegated the high importance, being intensely debated at the international forums organised by the United Nations, while there are visible the great progress in solving these problems. The main goal of food safety is to reduce the physical, chemical and biological risks that could cause the diseases among consumers (Aruoma, 2006).

\section{Methodology}

Methodological framework of the paper is based on the use of desk research, as well as descriptive and deduction method.

For a better understanding of functioning of the mechanisms within the agri-food sector regarding the use of chemically synthesized substances administered in animal husbandry, specifically in order to identify ways and possibilities to reduce the risk of human contamination with antibiotics residues in food products, conducted analysis was based on available data provided by FAO, EFSA, WHO. Besides, analysis has been involved consultation of adequate national and international scientific and professional literature oriented to the link between the use of antibiotics in animal husbandry and food safety.

The main subject of the paper is to determine the risks related to antibiotics administration in animal husbandry on human health. Paper aims to identify ways and possibilities to reduce the negative effects of animal medicaments treatments through responsible use. In order to achieve the main objective, conducted analysis tries to reach each production stage from farm to fork.

\section{Results and Discussion}

First part of the research is carried out along the importance of responsible use of drugs in animal husbandry related to environment protection and human health. This 
is the main rule of a sustainable food industry and represents a desideratum for EFSA (European Food Safety Authority). Even if the antibiotics residues are not correctly labelled in the food market, they exist and represent a real problem for human health due to their novice character.

\section{Responsible use of antibiotics}

The livestock, poultry and aquaculture sectors have always faced new challenges in terms of animal welfare and health, which, in addition to paying special attention to water and feed, require the administration of drug treatments against pathogenic bacteria that proliferate rapidly and strongly. As they are used more often and in larger quantities, antibiotics lose their effectiveness both in human medicine and livestock farming. So, responsible use is the key factor in maintaining the efficiency of antibiotics against microbial agents. Responsible use of antibiotic-based treatments requires adherence to a good practice guide that each production/processing unit must consider. Mentioned involves:

1. Use of antibiotics only if it is appropriate and in the right context.

2. Use of individual animals' treatment depends on their needs.

3. Administration of antibiotics in animal husbandry will be done only on the basis of a veterinary prescription.

4. Optimization of preventive and corrective measures is absolutely necessary, such as ensuring compliant shelters, hygiene programs, air conditioning, nutrition and monitoring programs for animal health and welfare. These conditions should always be met before starting antibiotics

According to a recent report of the European Parliament's Environment Committee, veterinary medicines (including antibiotics) should not be used to improve the economic performance of farms or to compensate for non-compliance with good animal husbandry practices. The responsible use of antibiotics is a good solution to reduce the risk of contaminated food. Any drug treatment must be verified and approved by a veterinary. Preventive administration of antimicrobial substances without clinical signs of infection presents a high risk of contamination of both herd and human population by increasing the resistance of bacteria to disease. On the other hand, when an animal in certain group shows specific symptoms of infection, treatment should be limited to this as well as to healthy animals, but identified as having a high risk of contamination, in order to prevent the further spread of bacteria within the herd. 


\section{Antibiotic residues and choices in the marketplace}

In the first stage of the agri-food production chain (at the farm level) a lot of substances are administered in order to protect plants and animals against diseases and pests. Every infectious disease, as well as every parasite or virus, is characterized by an antibiotic substance against them. Preventive measures often involve the administration of these treatments, without showing clinical signs of disease at the certain plant or animal. This procedure is not only unnecessary but it is also dangerous, as the body of animals accumulates a large amount of chemicals from the specific treatments with hormones, antibiotics and other veterinary drugs. All these substances finally end in meat, milk, eggs, honey, while then they have been consumed by human population, increasing their resistance to microbial agents.

As it can be seen in Figure 1., the road of antibiotics from farm to fork is very short and easy to trace. During the each stage of production chain, raw material becomes more contaminated, eventually accumulating all substances, impurities and bacteria on the contact surfaces during processing, transport, storage or handling by uninformed or non-compliant personnel for hygiene and food safety measures. Discussion about the risks that can occur in each stage of production, distribution and marketing of agri-food products is very long and includes many aspects to consider. An effective management system that will identify and mitigate the risks, regulate air and water circulation in production or processing facilities, adequate manure management and avoiding the soil pollution with contaminated agents is essential for meeting the standards and ensuring the food safety.

Currently, the agri-food market is showing such an unprecedented supply that consumers are sometimes disoriented due to the wide variety of products. They should make conscious choices based on the way how the animals were raised on the farm and what chemical residues can be found in the purchased animal products. But this goal is sometimes difficult to be achieved as the used labelling system in market is not always efficient system that can correctly and sufficiently inform the final consumer. For example, the label does not include the amount of chemical residues that may be found in the tissues of animal products. On the other hand, if the product is not correctly labelled, a majority of consumers do not know the effects of the chemical compounds and additives inscribed, while their choice to buy a product is usually unconscious. Good solution to this shortcoming is the introduction of a subject on food ethics in the school curriculum, explaining what information are the most valuable in this case. 
Figure 1. How antibiotics end up causing chemical residues and increasing the risk of human exposure

\section{START}

administration of

antibiotics in animal

Antibiotics are used more to prevent

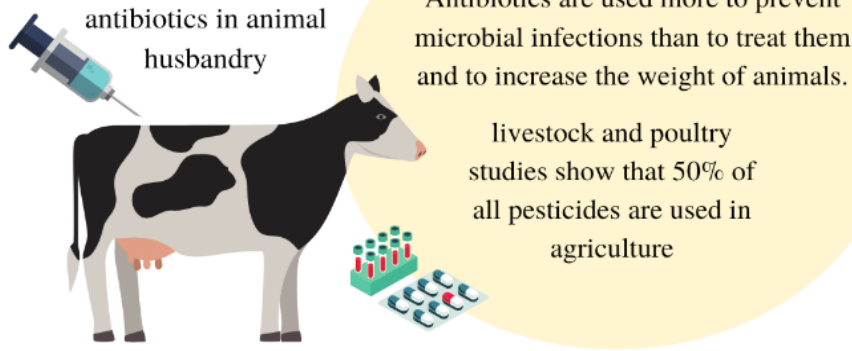

Once ingested, antibiotics produce resistance in the body of animals where bacteria are no longer threatened and can grow and proliferate exponentially.

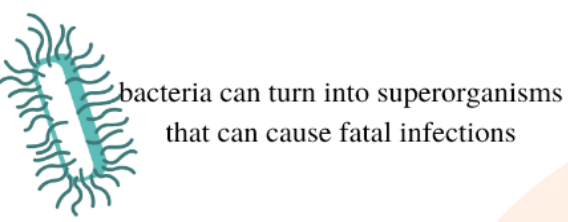

Bacteria that have already mutated and are resistant to antibiotics are transmitted to humans and their bodies will not be able to resist even common infections.

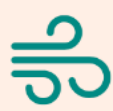

air

The administration of antibiotics determines the appearance of chemical residues in the finished products and these are transmitted through:

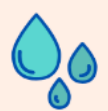

water

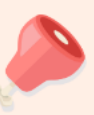

meat

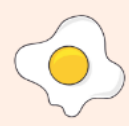

eggs, milk,

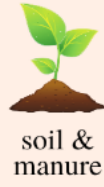

manure

thus, man is continuously exposed to antibiotics and their residues

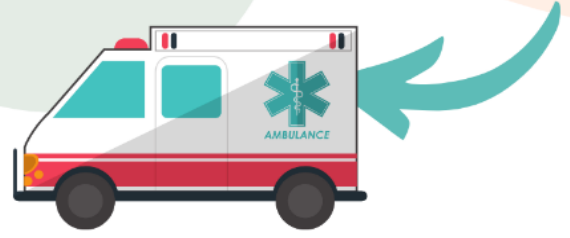

Source: According to authors opinion. 


\section{Current situation regarding livestock and poultry}

The analysis of statistical data on the use of antibiotics and drug treatments in animal husbandry and the pharmaceutical industry will create an overview of phenomena manifested in recent years on the meat products market and will help to identify potential risks of contamination. Analysis is carried out along with a study based on statistical data provided by EFSA, MEPs (Members of the European Parliament) and Consumers International. It was tried to highlight some important facts that can lead to a better understanding of how and why the antibiotics are used in livestock and poultry:

1. According to joint analysis of the European Food Safety Authority (EFSA), European Centre for Disease Prevention and Control (ECDC) and European Medicines Agency (EMA), drug treatments based on the administration of antibiotics are generalized and used in proportion of $91 \%$ for the entire herd and only $9 \%$ in individual treatment (ECDC/EFSA/EMA, 2017). Analysis of mentioned practice used by veterinarians and animal breeders materializes in negative externalities on human exposure to chemical residues of antibiotics in consumed meat, although the generalized treatment will not be effective against all bacteria. Each pathogenic microorganism develops and depends on environmental conditions, being characterized by different resistance to antibiotics, requiring the individual treatment. While some pathogenic bacteria are naturally resistant to certain types of antibiotics, others once exposed to these substances, begin to use the best defence mechanisms and thus become more resistant.

2. According to a recent report by Consumer International (2017), about $50 \%$ of antibiotics synthesized worldwide are used in animal husbandry and aquaculture. In quantifiable terms regarding the risk of human exposure to these substances, the percentage is enormous, especially since once used in fish and animal farms, antibiotics certainly reach the consumer's table. In order to reduce this number, the most common fast-food restaurants at 250,000 locations in the United States start to change the current situation by providing "cleaner" meat products with a lower risk of contamination. In this sense, continuous monitoring and control at each stage of the production process are absolutely necessary so that the final product does not exceed the allowable limit of chemical residues from the use of antibiotics in livestock and poultry.

3. Several studies conducted by the MEP (Grossetete et al., 2016) show that irresponsible use of antibiotics not only causes risks of contamination with substances useless to a healthy organism but will also cause considerable 
economic losses due to increased resistance of bacteria and thus their proliferation among animals and humans. If these pathogenic microorganisms can no longer be controlled by basic treatments, they will cause more deaths than cancer in upcoming decades. Some estimation shows that antibiotic use will increase for $60 \%$ until 2030.

4. Drug treatments based on the administration of antibiotics in livestock and poultry production are not only used to prevent certain bacterial infections. They also have the role to stimulate the muscle growth by acting in synergy with hormone-based treatments. In line to information of the Consumer International (2017), increasing the resistance of pathogenic bacteria generates major economic losses, with annual medical costs of up to 20 million USD.

\section{Maximum limit of medicinal residues allowed for human consumption}

According to the European Commission, all production and processing units in the agri-food sector are required to perform laboratory tests to detect the amount of chemical residues from the use of drug treatments and growth hormones at animal farms, verifying that they are below the $100 \mathrm{mg}$ threshold per $\mathrm{kg}$ of product (EC, 2019). In order to avoid contamination of food of animal origin, it is recommended to observe exactly the critical control limits present in the HACCP system of each unit, and if this limit is exceeded to apply corrective measures such as: withdrawal of contaminated food from stock, decontamination of food surfaces, application of basic principles (air circulation, protection of employees, avoidance of the use of specific chemical agents such as detergents, chemical cleaning substances, etc.), etc. Veterinary practice includes the intensive use of drug treatments based on sulphonamides, which causes contamination of the entire food chain from the farm to the fork with chemical residues.

In 2002, the European Commission validated some criteria as the methods of analysis for prevention: limit of detection and quantification, accuracy, specificity, accuracy, linearity, also serving from an economic point of view because it is a fast and cheap method. The methods most often used to detect chemical residues of sulphonamides in biological material are: immunosorbent enzyme assay, high performance liquid chromatography, mass spectrometry, determination of immune-biosensors and high performance capillary electrophoresis. These methods are quite expensive for Romanian specialists, which usually use instead the microbiological methods, as in that case specialized equipment is not required. Micro-bacterial substances within the sulphonamide class are widely used to maintain the health of the herd, as their application is ease, they are 
effective and cheap. Unfortunately, exceeding the maximum limit of chemical residues is found worldwide with a very high incidence among pigs, cattle and poultry. The danger of ingesting food contaminated with sulphonamides lies in the inability to comply with food safety principles, as studies show that very small amounts (less than $1 \mathrm{~g}$ of sulfamethazine per ton of food) can leave the residues in the human liver and muscles. Continuous monitoring of the agrifood chain and each stage within the chain of animal farming, since 2007 have been mandatory condition regulated by the presence of critical control limits imposed by the Codex Alimentarius Commission and the European Agency for Veterinary Medicine.

\section{Conclusions}

Responsible use of treatments based on the administration of antibiotics in the livestock sector, and continuous development of research on discovering new alternatives to replace old veterinary practices are necessary for the sustainable development of livestock production. In order to prevent and minimize the risks caused by the increased resistance of microbial agents toward the antibiotics, the livestock sector must be deeply and continuously monitored. In same time the antibiotics must be administered individually and in line to requirements derived from the diagnosis for each animal in the herd. The potential risk of irresponsible antibiotic administration is a widely debated issue due to decision makers' effort to enforce the law and determine a safe amount of drug treatment for each herd. In order to maintain a good wealth for animals, an effective management system must contain certain rules as: constant monitoring, effective hygiene and feed management, responsible use of antibiotics, hormones and other drugs, etc.

\section{References}

1. Aarestrup, F. M., Bager, F., Jensen, N. E., Madsen, M., Meyling, A., Wegener, H. C. (1998). Surveillance of antimicrobial resistance in bacteria isolated from food animals to antimicrobial growth promoters and related therapeutic agents in Denmark. APMIS, 106(1-6):606-622.

2. Aruoma, O. I. (2006). The impact of food regulation on the food supply chain. Toxicology, 221(1):119-127.

3. Auler, D., Teixeira, R., Nardi, V. (2017). Food safety as a field in supply chain management studies: A systematic literature review. International Food and Agribusiness Management Review, 20(1):99-112. 
4. CDC (2013). Antibiotic resistance threats in the US - 2013. Centers for Disease Control and Prevention (CDC). Atlanta, USA, retrieved at: www.cdc.gov/ drugresistance/pdf/ar-threats-2013-508.pdf, 24 ${ }^{\text {th }}$ October 2020.

5. Consumer International (2017). Get Antibiotics off the menu. Portal of the Consumers International, London, UK, retrievedat: www.consumersinternational. org/take-action/antibiotics-off-the-menu, $16^{\text {th }}$ October 2020.

6. Dani, S., Deep, A. (2010). Fragile food supply chains: reacting to risks. International Journal of Logistics: Research and Applications, 13(5):395-410.

7. ECDC/EFSA/EMA (2017). ECDC/EFSA/EMA second joint report on the integrated analysis of the consumption of antimicrobial agents and occurrence of antimicrobial resistance in bacteria from humans and food-producing animals (Joint Interagency Antimicrobial Consumption and Resistance Analysis (JACRA). EFSA Journal, 15(7):4872, https://doi.org/10.2903/j.efsa.2017.4872

8. EC (2019). Regulation (EU) 2019/1715, about Information management system for official controls to ensure compliance with agri-food chain rules, European Commission, Brussels, Belgium.

9. FAO (2003). The state of food insecurity in the World: 2003. Food and Agriculture Organization of the United Nations (FAO), Rome, Italy, retrieved at www.fao. org/3/j0083e/j0083e00.htm, $26^{\text {th }}$ October 2020.

10. Grossetete, F., Tanasescu, C., Piecha, B., Federley, F., Kyllonen, M., Hausling, M., Pedicini, P., Muller, U. (2016). Superbugs: MEPs want to curb use of antibiotics in farming. Portal News of the European Parliament, Brussel, Belgium, retrieved at: www.europarl.europa.eu/news/en/press-room/20160303IPR16930/superbugsmeps-want-to-curb-use-of-antibiotics-in-farming, $27^{\text {th }}$ October 2020.

11. Kuei, C. H., Madu, C. N., Lin, C. H. (2011). Developing global supply chain quality management systems. International Journal of Production Research, 49(15):4457-4481.

12. Lin, J. (2014). Antibiotic growth promoters enhance animal production by targeting intestinal bile salt hydrolase and its producers. Frontiers in Microbiology, 5(33):1-4, doi:10.3389/fmicb.2014.00033

13. Millet, S., Maertens, L. (2011). The European ban on antibiotic growth promoters in animal feed: From challenges to opportunities. Veterinary Journal, 187(2):143144, doi:10.1016/j.tvj1.2010.05.001

14. Roth, A. V., Tsay, A. A., Pullman, M. E., Gray, J. V. (2008). Unraveling the food supply chain: strategic insights from China and the 2007 recalls. Journal of Supply Chain Management, 44(1):22-39. 
15. Soltani, M., Alimardani, R., Omid, M., Karaj, I. (2011). Changes in physicomechanical properties of banana fruit during ripening treatment. Journal of American Science, 7(5):5-10.

16. Stanton, T. B. (2013). A call for antibiotic alternatives research. Trends in Microbiology. 21(3):111-113, doi: 10.1016/j.tim.2012.11.002

17. WHO (2019). Food safety: Key facts. World Health Organization (WHO), Geneva, Switzerland, retrieved at: www.who.int/news-room/fact-sheets/detail/ food-safety, $25^{\text {th }}$ October 2020. 